\title{
Vitamin D status in pre-school children in rural Nepal
}

\author{
Diana Avagyan', Sudan Prasad Neupane ${ }^{2}$, Thomas E Gundersen ${ }^{3}$ and \\ Ahmed A Madar ${ }^{1, *}$ \\ 'Department of Community Medicine, Institute of Health and Society, University of Oslo, Postboks 1 130, Blindern \\ 0318 Oslo, Norway: ${ }^{2}$ Norwegian Centre for Addiction Research (SERAF), Institute of Clinical Medicine, University \\ of Oslo, Oslo, Norway: ${ }^{3}$ Vitas Analytical Laboratory, Oslo Innovation Park, Oslo, Norway
}

Submitted 6 July 2014: Final revision received 26 January 2015: Accepted 4 February 2015: First published online 1 April 2015

\begin{abstract}
Objective: Vitamin D plays a major role in $\mathrm{Ca}$ and bone metabolism, and its extraskeletal functions are being appraised. Although inadequate vitamin D concentrations have been reported in populations worldwide, too little is known about vitamin D status and its determinants among children in developing countries. We aimed to determine vitamin D status and its determinants in Nepalese children of pre-school age.

Design: A community-based, cross-sectional study.

Setting: Rural Nepal at latitude $27 \cdot 39^{\circ} \mathrm{N}$.

Subjects: Healthy children ( $n$ 280) aged 12-60 months, selected randomly from the records of a vitamin A supplementation programme. Blood samples were collected using the dried blood spot technique and analysed for serum 25hydroxyvitamin D (s-25(OH)D) concentration using liquid chromatographytandem mass spectrometry. Ca intake and background variables were assessed with a structured questionnaire.

Results: Hypovitaminosis D, defined as s-25(OH)D concentration less than $50 \mathrm{nmol} / \mathrm{l}$, was found in $91.1 \%$ of the children. S-25(OH)D concentration was not related to gender, socio-economic indicators, sun exposure or nutritional status. Currently breast-fed children had higher s-25(OH)D concentrations (36.4 (SD 13.2) $\mathrm{nmol} / \mathrm{l})$ than those who were not (28.6 (sD 9.8) nmol/1, $P<0.001)$. Adjustment for sociodemographic factors did not alter the results.

Conclusion: There is widespread vitamin D deficiency among pre-school children in a rural area of Nepal. In our sample, sociodemographic factors did not affect the vitamin D status of children, but prolonged breast-feeding was associated with higher s-25(OH)D concentrations. Further research is required to investigate the health consequences of poor vitamin D status for this population.
\end{abstract}

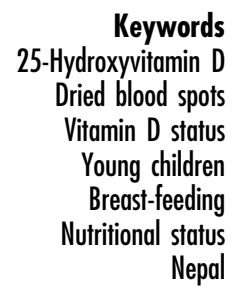

Low levels of vitamin D are reported in diverse populations around the world ${ }^{(1-3)}$, with one review suggesting that as many as $14 \%$ of the world's population have levels inadequate for general well-being ${ }^{(4)}$. Serum concentration of 25-hydroxyvitamin D $(\mathrm{s}-25(\mathrm{OH}) \mathrm{D})$ is the standard assessment for vitamin D status in the body and s-25(OH)D concentration of $50 \mathrm{nmol} / 1$ or more is considered sufficient for optimal bone-related health outcomes by the Institute of Medicine ${ }^{(5)}$.

Studies from Turkey, the Middle East and India have revealed that up to $50 \%$ of children have vitamin $\mathrm{D}$ deficiency $^{(6)}$. A number of determinants that affect s-25(OH)D concentration in children have been identified and include skin pigmentation, prolonged breast-feeding without vitamin D supplementation and low consumption of foods rich in vitamin $\mathrm{D}^{(7)}$. However, little is known about the vitamin D status of Nepalese children.

Nepal is a predominantly Hindu nation bordering Tibet and India, located between the latitudes of $26^{\circ} \mathrm{N}$ and $31^{\circ} \mathrm{N}$. Nepalese cultural values do not restrict sun exposure and about $300 \mathrm{~d}$ per year are sunny ${ }^{(8)}$. A few studies that have investigated vitamin D status in special groups in Nepal, such as pregnant women and alcohol-abusing populations, report a wide range of vitamin $\mathrm{D}$ deficiency prevalence rates $(4-64 \%)^{(9,10)}$. One recent study found $19 \%$ of pre-adolescent Nepalese children to have vitamin D deficiency ${ }^{(11)}$. The samples were taken year-round and no assessment of clothing habits or dietary intake was conducted, which makes the findings difficult to interpret. Children under 5 years of age are particularly vulnerable to 
different infections and have greater mineral demands due to their growing bones; however, there are no data on vitamin D status in this age group in the South Asian region. Thus, we sought to determine the vitamin D status among children aged 12-60 months in rural Nepal and to identify its possible associations with sociodemographic indicators, breast-feeding practices and nutritional status.

\section{Methods}

\section{Study population}

The present community-based, cross-sectional study was conducted in rural Nepal, at latitude of $27 \cdot 39^{\circ} \mathrm{N}$ and during the dry season, between September and November 2012. Children from 12 to 60 months of age were recruited from the nine wards of Ugrachandi Nala Village Development Committee in Kavrepalanchowk district of Central Nepal. We utilized the existing national vitamin A supplementation programme that maintains a national registry of children in this age group ${ }^{(12)}$. From the records available at the local health post, we assigned numbers to all 411 children aged 12-60 months living in the target village. By using an online randomizer, a set of 320 uniquely sorted numbers was generated to randomly select the participants. Female community health volunteers were requested to identify the selected children and invite their guardians for participation in the study. None of the children we screened had known metabolic bone disease or chronic disease associated with bone abnormalities, and none used medications likely to interfere with vitamin D metabolism. A total of 280 participants, all of whom provided complete questionnaires, were included in our analysis.

\section{Collection of background information}

The guardians of each child provided sociodemographic information, including information about family size, occupation and household income. Information on breastfeeding practices, consumption of milk products, use of vitamin supplements, medical history and sun exposure was also collected by a trained nurse from the same area, using a structured questionnaire. Household income and type of housing (made from natural or manufactured materials) provided proxy measures of socio-economic status. Pre-testing of the questionnaire among mothers from the same setting showed that foods naturally rich in vitamin D did not constitute the dietary supply in general. Therefore, dietary assessment of vitamin D-providing foods was not conducted.

Milk product intake was calculated by multiplying the amount per serving by the food frequency for each type of milk product. Ca intake was computed by multiplying the Ca content of the specific type of milk product by the daily consumed amount. Total $\mathrm{Ca}$ intake from milk products was calculated by summing the Ca intake from different milk products for each child. Ca values in milk products were obtained from the US Department of Agriculture's National Nutrient Database for Standard Reference ${ }^{(13)}$. Weight was measured with a digital weight scale made by Microlife Company in Switzerland, certified for ISO 9001 with accuracy of $100 \mathrm{~g}$. Height was measured upright, using a wall stadiometer (Bio-Plus $200 \mathrm{~cm}$, model $265 \mathrm{M} / 1013522$ ). Standing height for children younger than 24 months was equalized to the recumbent length by adding $0.7 \mathrm{~cm}$. All measurements were taken without shoes and with minimal clothing.

\section{Assessment of nutritional status}

The nutritional status of the children was determined by comparing measurements of weight-for-height, weightfor-age and height-for-age with the WHO reference standards for wasting, underweight and stunting, respectively $^{(14)}$. Weight-for-height (indicator of wasting, i.e. acute or recent malnutrition), weight-for-age (indicator of underweight, i.e. acute or chronic malnutrition) and height-for-age (indicator of stunting, i.e. chronic or longstanding malnutrition) were defined according to WHO standards ${ }^{(15)}$.

\section{Dried blood spot sampling and vitamin $D$ assay}

Capillary blood was collected by a certified nurse after pricking the fingertip with a single-use safety lancet. The total amount collected was approximately 4-5 drops. Blood drops were applied directly on the sampling filter card within pre-marked circles. The cards were then air dried for $2 \mathrm{~h}$; sample cards were stored in low gas-permeable zip-lock bags with desiccant packages. Samples were kept at room temperature during the first $7 \mathrm{~d}$, and later refrigerated. At the end of sample collection, the dried blood spot (DBS) kits were shifted to the laboratory of Vitas AS in Norway by courier service for further analysis. Serum concentrations of 25-hydroxycholecalciferol $\left(\mathrm{s}-25(\mathrm{OH}) \mathrm{D}_{3}\right)$ and 25-hydroxyergocalciferol (s-25(OH)D $\left.\mathrm{D}_{2}\right)$ were quantified separately using liquid chromatographytandem mass spectrometry (LC-MS/MS). S-25(OH)D was expressed as the sum of $s-25(\mathrm{OH}) \mathrm{D}_{2}$ and $s-25(\mathrm{OH}) \mathrm{D}_{3}$. Punches from the DBS were added to water, shaken and diluted with 2-propanol-methanol containing the internal standard $\left[{ }^{2} \mathrm{H}\right]_{6}-25(\mathrm{OH}) \mathrm{D}_{3}$ and butylated hydroxytoluene as an antioxidant. After mixing and centrifugation the supernatant was transferred to an insert and centrifuged again, and an aliquot of $50 \mu \mathrm{l}$ was injected into the HPLC system. HPLC was performed with an Agilent 1200 liquid chromatograph (Agilent Technologies, Palo Alta, CA, USA) interfaced by atmospheric pressure chemical ionization to an Agilent mass spectrometric detector operated in Multiple Reaction Monitoring mode. Vitamin D analogues were separated on a $4.6 \mathrm{~mm} \times 150 \mathrm{~mm}$ reversed-phase column with $2 \cdot 7 \mu \mathrm{m}$ particles. The column temperature was $40^{\circ} \mathrm{C}$. A two-point calibration curve was made from analysis of DBS calibrators with known vitamin D concentrations. The LC-MS/ MS DBS method was internally validated. The inter- and 
intra-assay $\mathrm{CV}$ were $7.6 \% \quad(35.8 \mathrm{nmol} / \mathrm{l})$ and $10.2 \%$ $(78.3 \mathrm{nmol} / \mathrm{l})$, respectively.

The LC-MS/MS DBS method was chosen as a minimally invasive and convenient to use technique for paediatric research participants. The laboratory performing the analysis is part of the Vitamin D Quality Assessment Scheme and is compliant.

A common universal normal range for s-25(OH)D does not exist; however, we chose to use the commonly used cut-off points, where vitamin D status is classified as severely deficient $(0-12.5 \mathrm{nmol} / \mathrm{l})$, moderately deficient (12.6-25.0 nmol/1), mildly deficient (25.1-49.9 nmol/1) and sufficient $(\geq 50 \cdot 0 \mathrm{nmol} / \mathrm{l})^{(16)}$.

\section{Etbical considerations}

After explaining the study aims and data collection procedure, consenting guardians were asked to provide written or witnessed verbal consent. The guardians were also the informants. Verbal consent was acquired when guardians had a decision-making capacity but could not read or write due to illiteracy; in these cases, verbal consent was witnessed by an adult from the same community who was not a member of the study team and formally recorded. The study was conducted according to the guidelines provided in the Declaration of Helsinki and all procedures involving human subjects/patients were approved by the Nepal Health Research Council and the Regional Committee for Medical and Health Research in Norway. Written or verbal, voluntary informed consent was obtained from all subjects/patients.

\section{Statistical analysis}

WHO Anthro software version 3.2.2 was used to evaluate sex-specific growth indicators according to WHO standards $^{(14)}$. Descriptive statistics are presented as means and standard deviations. To compare the mean s-25(OH)D concentrations according to explanatory variables, we used an independent-sample $t$ test (two-tailed) and one-way ANOVA. The relationships between s-25(OH)D and potentially associated variables were tested primarily using linear regression models, both for continuous and categorical explanatory variables. Assumptions of the regression analysis (linearity and equal variance for the dependent variable) were checked by inspecting plots of residuals against predicted values. Statistical analysis of the data was performed using the statistical software package IBM SPSS Statistics version 20. Significance level was set to $0 \cdot 05$.

\section{Results}

\section{Sample characteristics}

A total of 280 children aged 12-60 months, mean age 35.8 (SD 13.8) months, were included in the study. Most children ( $81 \cdot 1 \%)$ from our sample (157 boys and 123 girls) were from low to medium socio-economic groups. The majority of parents (98\%) reported trousers and longsleeved shirts as regular clothing for their children; $16 \%$ mentioned T-shirts as an option for warm days. In our sample, exclusive breast-feeding during the first 6 months was universal (99.3\%). The mean duration for breastfeeding was estimated to be 27 (SD 7) months. Moreover, $38 \%$ of the mothers reported that their children were still breast-feeding. The total Ca intake for all the children was 318 (sD 235) $\mathrm{mg} / \mathrm{d}$. About $45 \%$ of the children were classified as stunted, $6 \%$ as wasted and $24 \%$ as underweight (Table 1).

\section{Vitamin D status}

The mean concentration of total s-25(OH)D was 31.5 (SD $12 \cdot 0) \mathrm{nmol} / 1$ for all infants, ranging between 6.9 and $74.5 \mathrm{nmol} / \mathrm{l}$. S-25(OH)D 2 concentration was detected only in nine children, with a mean of 8.4 (SD 5.6) nmol/1. As shown in Table 2, over $90 \%$ of the children had s-25(OH)D concentrations below $50 \mathrm{nmol} / \mathrm{l}$, whereas about a third of the children had s-25(OH)D concentrations in the moderate to severe deficiency range and only $9 \%$ had sufficient s-25(OH)D concentrations.

Table 1 also shows s-25(OH)D concentrations according to the different sociodemographic and nutritional categories. There was no difference in mean s-25(OH)D concentrations between boys and girls. Younger children (12-24 months) had higher s-25(OH)D concentrations than older children $(P<0 \cdot 01)$. The mean concentration of $\mathrm{s}-25(\mathrm{OH}) \mathrm{D}$ was $36.4(\mathrm{sD} 13 \cdot 2) \mathrm{nmol} / \mathrm{l}$ and 28.6 (sD 9.8) $\mathrm{nmol} / \mathrm{l}$ for children younger than 36 months who were currently breast-fed and not currently breast-fed, respectively. In linear regression models analysing the relationship between s-25(OH)D and potentially associated factors in Nepalese children, s-25(OH)D concentration was significantly higher among children who were currently breast-fed compared with those not currently breastfed $(P<0 \cdot 001)$. Children living in mud/wooden houses had higher $\mathrm{s}-25(\mathrm{OH}) \mathrm{D}$ concentrations than those living in concrete houses ( $P$ 0.048). Other variables such as nutritional status, household income, time spent outdoors daily and $\mathrm{Ca}$ intake were not associated with s-25(OH)D concentrations (Table 3).

\section{Discussion}

We found that vitamin D deficiency is common among children in rural Nepal, with over $90 \%$ of children $1-5$ years of age having s-25(OH)D concentrations under $50 \mathrm{nmol} / \mathrm{l}$. Severe vitamin D deficiency $(<12.5 \mathrm{nmol} / \mathrm{l})$ was found in $4 \%$ of the studied children. There are limited data on vitamin D status among children of pre-school age in the South Asian region; however, the findings of the current study are consistent with the few reports of vitamin D status among healthy pre-school children ${ }^{(17,18)}$. Using the cut-off concentration of $50 \mathrm{nmol} / \mathrm{l}$, Wayse et al. reported 
Table 1 Demographic and nutritional characteristics and s-25(OH)D concentrations (nmol/l) of rural Nepalese children $(n 280)$ aged 12-60 months, September-November 2012

\begin{tabular}{|c|c|c|c|c|c|}
\hline & \multicolumn{2}{|c|}{ Characteristic } & \multicolumn{2}{|c|}{$\mathrm{s}-25(\mathrm{OH}) \mathrm{D}$} & \multirow[b]{2}{*}{$P$ value } \\
\hline & $n$ & $\%$ & Mean & SD & \\
\hline \multicolumn{6}{|l|}{ Age (months) } \\
\hline $12-24$ & 93 & 33.2 & 35.4 & 13.1 & \\
\hline $25-36$ & 72 & $25 \cdot 7$ & 30.8 & 11.4 & \\
\hline $37-60$ & 115 & $41 \cdot 1$ & $29 \cdot 0$ & 10.7 & 0.01 \\
\hline \multicolumn{6}{|l|}{ Sex } \\
\hline Female & 123 & 43.9 & 31.2 & $11 \cdot 2$ & \\
\hline Male & 157 & $56 \cdot 1$ & 31.9 & $12 \cdot \overline{6}$ & 0.6 \\
\hline \multicolumn{6}{|c|}{ Household income (monthly, NRS*) } \\
\hline Below 14999 & 140 & $50 \cdot 0$ & $32 \cdot 7$ & $12 \cdot 2$ & \\
\hline $15000-39999$ & 87 & $31 \cdot 1$ & 31.1 & 11.7 & \\
\hline Above 40000 & 53 & $18 \cdot 9$ & $29 \cdot 4$ & 11.9 & 0.2 \\
\hline \multicolumn{6}{|c|}{ Currently breast-feeding (for children aged $12-36$ months) } \\
\hline Yes & 102 & $61 \cdot 8$ & $36 \cdot 4$ & 13.2 & \\
\hline No & 63 & $38 \cdot 2$ & 28.6 & $9 \cdot 8$ & 0.001 \\
\hline \multicolumn{6}{|l|}{ Nutritional status $†$} \\
\hline \multicolumn{6}{|l|}{ Weight-for-height } \\
\hline Corresponds to normal & 251 & $94 \cdot 4$ & $31 \cdot 2$ & 11.6 & \\
\hline Wasting $\ddagger$ & 15 & $5 \cdot 6$ & 38.2 & $15 \cdot 7$ & $0 \cdot 11$ \\
\hline \multicolumn{6}{|l|}{ Weight-for-age } \\
\hline Corresponds to normal & 214 & 76.4 & 31.1 & 11.7 & \\
\hline Underweight & 47 & $16 \cdot 8$ & 31.6 & 11.4 & \\
\hline Severe underweight & 19 & 6.8 & 37.5 & $15 \cdot 4$ & 0.98 \\
\hline \multicolumn{6}{|l|}{ Height-for-age } \\
\hline Corresponds to normal & 148 & $55 \cdot 4$ & 31.7 & $12 \cdot 2$ & \\
\hline Stunting & 74 & $27 \cdot 7$ & 31.0 & 11.8 & \\
\hline Severe stunting & 45 & $16 \cdot 9$ & $32 \cdot 8$ & 11.6 & 0.74 \\
\hline
\end{tabular}

s-25(OH)D, serum 25-hydroxyvitamin D.

*NRS, Nepalese Rupees; \$US 1=approx. NRS 100 as of 30 September 2012.

†Nutritional status defined according to WHO standards.

¥Nutritional status determined with cut-offs $-2 \mathrm{SD}$, includes three cases with score below $-3 \mathrm{sD}$.

Table 2 Vitamin D status on the basis of s-25(OH)D concentration among rural Nepalese children $(n$ 280) aged 12-60 months, September-November 2012

\begin{tabular}{lccc}
\hline & & \multicolumn{2}{c}{ Prevalence among study population } \\
\cline { 3 - 4 } & s-25(OH)D cut-off $(\mathrm{nmol} / \mathrm{l})$ & $\%$ & $n$ \\
\hline Sufficient & 250.0 & 8.9 & 25 \\
Mild deficiency & $25 \cdot 1-49.9$ & 61.8 & 173 \\
Moderate deficiency & $12 \cdot 6-25 \cdot 0$ & 25.4 & 71 \\
Severe deficiency & $0-12.5$ & 3.9 & 11 \\
\hline
\end{tabular}

S-25(OH)D, serum 25-hydroxyvitamin D.

vitamin D deficiency among $61 \%$ of Indian children under 5 years of age ${ }^{(17)}$. Roth et al. found a mean s-25(OH)D concentration of $39.2 \mathrm{nmol} / \mathrm{l}$ among Bangladeshi children aged 1-23 months ${ }^{(18)}$. Similarly high prevalence of vitamin D deficiency was identified among pregnant women and their newborns, adolescents and adults in the region ${ }^{(19-23)}$

\section{Factors associated with serum}

\section{5-bydroxyvitamin $D$ concentrations}

In contrast to findings from studies conducted among Indian children, which showed that boys had significantly higher s-25(OH)D concentrations than girls ${ }^{(20,24)}$, we did not observe any significant sex difference in vitamin D status among our Nepalese children. This could be explained by the similar clothing practices and outdoor activities of both sexes in our sample, as noted by Sahu et al. with regard to teenagers in rural India ${ }^{(24)}$. As compared with samples of Pakistani infants ${ }^{(25)}$ and Indian schoolgirls ${ }^{(21)}$, we did not observe differences in s-25(OH)D concentration with regard to socio-economic factors such as income level of the family. Nevertheless, children living in mud/wooden houses had slightly higher s-25(OH)D concentrations than those living in concrete houses. This difference disappeared, however, after we adjusted for breast-feeding practices because more children who were currently breast-fed lived in a mud/wooden 
Table 3 Relationship between s-25(OH)D (nmol/l) and potentially associated factors in rural Nepalese children ( $n$ 280) aged $12-60$ months, September-November 2012

\begin{tabular}{|c|c|c|c|c|c|c|}
\hline Explanatory variable & $\begin{array}{l}\text { Unadjusted } \\
\text { regression } \\
\text { coefficient }\end{array}$ & $95 \% \mathrm{Cl}$ & $P$ value & $\begin{array}{l}\text { Adjusted } \\
\text { regression } \\
\text { coefficient }^{\star}\end{array}$ & $95 \% \mathrm{Cl}$ & $P$ value \\
\hline Breast-feeding now $†$ & $7 \cdot 0$ & $4 \cdot 1,9 \cdot 8$ & $<0.001$ & 6.6 & $3 \cdot 1,10 \cdot 1$ & $<0.001$ \\
\hline Age in months $\ddagger$ & $-4 \cdot 4$ & $-7 \cdot 3,-1 \cdot 5$ & 0.003 & -0.7 & $-4 \cdot 1,2 \cdot 8$ & 0.7 \\
\hline Sex§ & -0.7 & $-3 \cdot 6,2 \cdot 2$ & 0.6 & -0.7 & $-3 \cdot 5,2.0$ & 0.6 \\
\hline Ca intake & 0.0 & $-0.007,0.005$ & 0.9 & 0.001 & $-0.005,0.007$ & 0.6 \\
\hline $\begin{array}{l}\text { Time spent outdoors } \\
\text { daily\|l }\end{array}$ & $-1 \cdot 1$ & $-4 \cdot 0,1 \cdot 8$ & 0.5 & $-0 \cdot 3$ & $-3 \cdot 1,2 \cdot 6$ & 0.8 \\
\hline Incomeף & $-1 \cdot 8$ & $-4 \cdot 7,1 \cdot 0$ & 0.2 & $-1 \cdot 0$ & $-3 \cdot 9,1 \cdot 8$ & 0.5 \\
\hline Type of housing ${ }^{\star \star}$ & $-3 \cdot 4$ & $-6 \cdot 7,-0 \cdot 1$ & 0.048 & $-2 \cdot 8$ & $-5.9,0.4$ & 0.09 \\
\hline
\end{tabular}

s-25(OH)D, serum 25-hydroxyvitamin D.

${ }^{*}$ Adjusted for variables that were significant in simple linear regression (breast-feeding now, type of housing and age).

†Breast-feeding now coded as: $0=$ no; $1=$ yes .

$\ddagger$ Age coded as: $1=12-36$ months; $2=37-60$ months.

$\S$ Sex coded as: 1 = boy; 2 = girl.

IITime spent outdoors coded as: $1=\leq 2 \mathrm{~h} ; 2=>2 \mathrm{~h}$.

IIncome coded as: $1=<$ NRS 15 000; $2=>$ NRS 15000 .

${ }^{\star *}$ Type of housing coded as: $1=$ mud and wood; $2=$ concrete.

house. In the Nepalese context, concrete housing is a marker of affluence, but at the same time may indicate decreased physical activity and limited opportunity for sun exposure. Findings from Neupane et al. ${ }^{(9)}$ suggested that rather than having a formal job, peasants who more often live in mud/wooden houses were more likely to have a better vitamin D status in Nepal. This factor as a potential predictor of vitamin D status remains to be tested specifically in different population groups.

High s-25(OH)D concentrations among low socioeconomic strata have previously been explained with reference to prolonged sun exposure due to outdoor occupation, as opposed to better household conditions and higher levels of education that may associated with restricted outdoor activities ${ }^{(21,25)}$. It seems for children of pre-school age in the present study that vitamin D status is not determined by socio-economic status because of similarly poor dietary supply of vitamin D and comparable exposure to sunlight across social strata.

In the literature, prolonged exclusive breast-feeding was reported as a risk factor for the development of hypovitaminosis $\mathrm{D}^{(26,27)}$. In our study, we tested whether prolonged breast-feeding was associated with vitamin D status. Surprisingly, we found that children who received breast milk in addition to traditional foods had better vitamin D status compared with those who did not. This difference was still significant when adjusted for age, household income, type of housing, daily time spent outdoors and $\mathrm{Ca}$ intake. This may be attributed not only to the nutritional component of breast-feeding but also to the practice itself. Given the fact that, in Nepalese culture, women breast-feed minimally clothed children outdoors and under the sun, we can assume that prolonged breastfeeding provides children with more opportunities for sun exposure. Also, younger children had significantly higher concentrations of $\mathrm{s}-25(\mathrm{OH}) \mathrm{D}$ than those in the older groups. Most of the children had poor growth and were malnourished; however, we did not find a significant difference in s-25(OH)D concentration when comparing by nutritional status of the children.

Ca intake calculated from milk and milk products was low $(318 \mathrm{mg} / \mathrm{d})$. During the data collection, it also was observed that children are quite often given cow or buffalo milk and homemade yoghurt, but the servings were very small.

Even though this finding coincides with similar studies conducted in the region, the reason for this high magnitude of poor vitamin D status remains unclear. In the literature, several factors have been postulated, such as skin pigmentation, traditionally poor diets with few natural sources of vitamin D, prolonged breast-feeding and unavailability of a national vitamin $\mathrm{D}$ supplementation programme $^{(1,28)}$.

\section{Limitations and strengths of the study}

The present study has several limitations. When selecting the population sample, randomization was applied. Due to the absence of demographic data in the region, we had to create our own databases from the vitamin A supplementation programme's demographic data available at the local health post. Although officially reported coverage for Nepal's national vitamin A supplementation programme for children aged 6-59 months was $90 \cdot 4 \%$ as of $2011^{(12)}$, it could be that those children who for some reason did not receive vitamin A supplements during last 6 months were not included in our databases and consequently excluded from selection potential for the current study. This kind of selection bias might suggest that our sample was more representative for those who have better access to healthcare programmes than for the general population. Apparently, Ca intake was not calculated accurately, as we used reported regular consumption of milk products and 
therefore cannot rule out recall bias; also, we did not account for other sources of dietary $\mathrm{Ca}$.

Despite these limitations, the study suggests a general pattern of vitamin D status among pre-school children in rural Nepal. Full sample size was achieved with a response rate of $87.5 \%$. The use of the DBS method is another strength and is a minimally invasive means of obtaining s-25(OH)D measurements, particularly in a rural setting without sample storage and transport possibilities. However, we should take into consideration that Nepal extends from less than 100 to $8850 \mathrm{~m}$ above sea level, which has an effect on vitamin D production throughout the whole year ${ }^{(29)}$. Therefore this result needs to be interpreted with respect to the study population and geographical coordinates.

\section{Conclusion}

We report a high prevalence of vitamin D deficiency among pre-school children in a rural area of Nepal at latitude $27.39^{\circ} \mathrm{N}$. In our sample, sociodemographic factors did not affect the vitamin D status of children, but prolonged breastfeeding was associated with better vitamin D status. Further research is required to investigate the health consequences of low vitamin D status for this population.

\section{Acknowledgements}

Acknowledgements: The authors are grateful to their research assistant, Pratibha Khatri, and to all community health volunteers from the Nala Ugrachandi Village Development Committee for their cooperation. They thank all the families and children who participated in the study. Financial support: This study was funded by the University of Oslo and supported in part by Vitas AS, Oslo, Norway for serum analysis. The funders had no role in the design, analysis or writing of this article. Conflict of interest: None. Authorship: D.A. and S.P.N. contributed equally to this work in terms of the analysis, interpretation and manuscript writing. D.A., S.P.N. and A.A.M. designed the study. D.A. carried out the fieldwork. D.A. and S.P.N. carried out the analysis and drafted the manuscript. T.E.G. performed laboratory analysis. A.A.M., T.E.G., D.A. and S.P.N. contributed to the interpretation of data, as well as critical revision of the manuscript. All authors approved the final version. A.A.M. is the guarantor of this work and, as such, had full access to all of the data in the study and takes responsibility for the integrity of the data and the accuracy of the data analysis. Ethics of human subject participation: The study was approved by the Nepal Health Research Council and the Regional Committee for Medical and Health Research in Norway. Written or verbal, voluntary informed consent was obtained from all subjects/patients.

\section{References}

1. Mithal A, Wahl DA, Bonjour JP et al. (2009) Global vitamin D status and determinants of hypovitaminosis D. Osteoporosis Int 20, 1807-1820.

2. Wahl DA, Cooper C, Ebeling PR et al. (2012) A global representation of vitamin D status in healthy populations. Arch Osteoporos 7, 155-172.

3. Lips P (2010) Worldwide status of vitamin D nutrition. J Steroid Biochem Mol Biol 121, 297-300.

4. Holick MF (2008) Vitamin D: a D-Lightful health perspective. Nutr Rev 66, 10 Suppl. 2, S182-S194.

5. Ross AC, Taylor CL, Yaktine AL et al. (2011) Dietary Reference Intakes for Calcium and Vitamin D. Washington, DC: National Academies Press.

6. El-Hajj Fuleihan G (2009) Vitamin D deficiency in the Middle East and its health consequences for children and adults. Clin Rev Bone Miner Metab 7, 77-93.

7. Babu US \& Calvo MS (2010) Modern India and the vitamin D dilemma: evidence for the need of a national food fortification program. Mol Nutr Food Res 54, 1134-1147.

8. World Energy Council and South Asian Association (2000) Renewable Energy in South Asia: Status and Prospects. London: World Energy Council.

9. Neupane SP, Lien L, Hilberg T et al. (2013) Vitamin D deficiency in alcohol-use disorders and its relationship to comorbid major depression: a cross-sectional study of inpatients in Nepal. Drug Alcohol Depend 113, 480-485.

10. Jiang T, Christian P, Khatry SK et al. (2005) Micronutrient deficiencies in early pregnancy are common, concurrent, and vary by season among rural Nepali pregnant women. J Nutr 135, 1106-1112.

11. Cole RN, Ruczinski I, Schulze K et al. (2013) The plasma proteome identifies expected and novel proteins correlated with micronutrient status in undernourished Nepalese children. J Nutr 143, 1540-1548.

12. Ministry of Health and Population, Government of Nepal, New ERA \& ICF International (2012) Nepal Demographic and Health Survey 2011. Kathmandu and Calverton, MD: Government of Nepal, New ERA and ICF International.

13. US Department of Agriculture, Agricultural Research Service (2013) USDA National Nutrient Database for Standard Reference. Beltsville, MD: USDA, ARS.

14. World Health Organization (2007) WHO Anthro for Personal Computers, Version 2, 2007: Software for Assessing Growth and Development of the World's Children. Geneva: WHO.

15. World Health Organization (2008) Training Course on Child Growth Assessment. Geneva: WHO.

16. Lips P (2004) Which circulating level of 25-hydroxyvitamin D is appropriate? J Steroid Biochem Mol Biol 89-90, 611-614.

17. Wayse V, Yousafzai A, Mogale K et al. (2003) Association of subclinical vitamin D deficiency with severe acute lower respiratory infection in Indian children under 5 years. Eur J Clin Nutr 58, 563-567.

18. Roth DE, Shah R, Black RE et al. (2010) Vitamin D status and acute lower respiratory infection in early childhood in Sylhet, Bangladesh. Acta Paediatr 99, 389-393.

19. Sachan A, Gupta R, Das V et al. (2005) High prevalence of vitamin $\mathrm{D}$ deficiency among pregnant women and their newborns in northern India. Am J Clin Nutr 81, 1060-1064.

20. Marwaha RK, Tandon N, Reddy DR et al. (2005) Vitamin D and bone mineral density status of healthy schoolchildren in northern India. Am J Clin Nutr 82, 477-482.

21. Puri S, Marwaha RK, Agarwal N et al. (2008) Vitamin D status of apparently healthy schoolgirls from two different socioeconomic strata in Delhi: relation to nutrition and lifestyle. Br J Nutr 99, 876-882. 
22. Marwaha RK, Tandon N, Chopra S et al. (2011) Vitamin D status in pregnant Indian women across trimesters and different seasons and its correlation with neonatal serum 25-hydroxyvitamin D levels. Br J Nutr 106, 1383-1389.

23. Goswami R, Gupta N, Goswami D et al. (2000) Prevalence and significance of low 25-hydroxyvitamin D concentrations in healthy subjects in Delhi. Am J Clin Nutr 72, 472-475.

24. Sahu M, Bhatia V, Aggarwal A et al. (2009) Vitamin D deficiency in rural girls and pregnant women despite abundant sunshine in northern India. Clin Endocrinol (Oxf) 70, 680-684.
25. Atiq M, Suria A, Nizami S et al. (1998) Vitamin D status of breastfed Pakistani infants. Acta Paediatr 87, 737-740.

26. Misra M, Pacaud D, Petryk A et al. (2008) Vitamin D deficiency in children and its management: review of current knowledge and recommendations. Pediatrics 122, 398-417.

27. Wagner CL \& Greer FR (2008) Prevention of rickets and vitamin D deficiency in infants, children, and adolescents. Pediatrics 122, 1142-1152.

28. Prentice A (2008) Vitamin D deficiency: a global perspective. Nutr Rev 66, 10 Suppl. 2, S153-S164.

29. Holick MF (2012) The D-lightful vitamin D for child health. JPEN J Parenter Enteral Nutr 36, 1 Suppl., 9S-19S. 